\title{
Development of MWCNTs/alumina composite tap by using gel cast technique for optical detector in the IR region: CNT concentration and light intensity dependent comparative study
}

\author{
Abid,RumysaManzoor, SamrahManzoor, Sami Ullah, NishithaSaxena, MohdTalib, \\ PoonamSehrawat and SS Islam*
}

Centre for Nanoscience and Nanotechnology, JamiaMilliaIslamia, Jamia Nagar, New Delhi-110025, India

*Email: sislam@jmi.ac.in

Carbon nanotubes have been the focus of study and research worldwide with respect to their sensitivity towards light and heat sources, gases, etc. thus they may be used to fabricate novel sensors and detectors which may render carbon nanotubes as 'Advanced Nano-materials' for sensor technology. Not much research has been done on the study of photo response of the CNTs in their pure and amorphous form.

The photoconductivity of

MWCNTs/Alumina composite tape has been studied under infrared illumination for different samples with varying concentration of CNT. The concentrations of CNT were taken as $0.6 \mathrm{wt} \%, 1 \mathrm{wt} \% 1.5$ $\mathrm{wt} \%, 2 \mathrm{wt} \%$, and $3 \mathrm{wt} \%$ and labeled as G1, G2, G3, G4, and G5. Surface morphology was investigated through FESEM and shown in fig 1(a). An IR laser with $1064 \mathrm{~nm}$ wavelength was used for the purpose. The photoconductivity was first observed for 3 seconds for each sample at $127 \mathrm{~mW} / \mathrm{mm}^{2}$ power density, which gave excellent response in the form of Resistivity Vs Time graph as shown in fig 1(b) and hence, the response time, recovery time, and sensitivity were calculated. The peaks observed were sharp with fast response time of about $2 \mathrm{~s}$ and the responsivity increases as $\mathrm{CNT}$ concentration increases till $2 \mathrm{wt} \%$ after that it start decreasing due to the formation of agglomeration of CNTs in the network or may be due the defects in the network which trap the electrons that are generated by the illumination of IR radiation. The average recovery time is of the order of 4 s. After analyzing the photoconductive response for 3 seconds, the samples were illuminated with the IR light at intensities of $64,80,95,127 \mathrm{~mW} / \mathrm{mm}^{2}$ for the same time period. The expected results were an increase in the resistance change with time or sensitivity with increasing intensities. Sample with $2 \mathrm{wt} \%$ CNT shows maximum sensitivity of about $32 \%$ but after that it starts decreasing due to the elimination of the dispersed nature of the CNTs in the films. The comparative study done on the response and recovery, and sensitivity of the different samples for different intensities of light is thus presented here in this paper.

The remarkable and novel features of this work are its fast response and recovery time and excellent reproducibility with negligible drift.
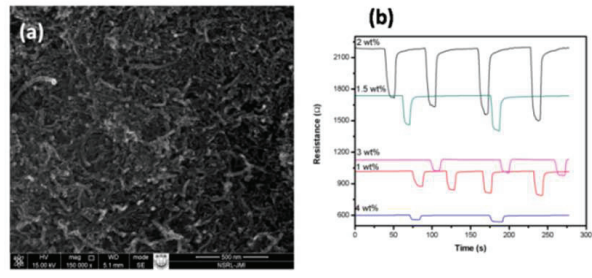

Figure 1: (a) FESEM image of G3 sample, and (b) photoresistive response of $\mathrm{G} 1, \mathrm{G} 2$, G3, G4 and G5. 